\title{
Racial Differences on Post Exercise Heart Rate Recovery in Healthy Sedentary Adult Males-- A Meta-Analysis Study
}

\author{
Sana Soomro*, Kausar Abbas Saldera, Ufaque Batool, Raheela Manghi \\ Department of Physiology, Basic Medical Science Institute, Jinnah Postgraduate Medical Center, \\ Karachi, Pakistan
}

Received: July 11, 2021 Accepted: December 30, 2021 DOI: $10.46568 /$ bios.v2i2.41

\begin{abstract}
:
Introduction: Post-exercise heart rate recovery (PEHRR) is the measure of parasympathetic function of heart which decreases the heart rate as the person stops exercising. The post exercise heart rate recovery is easily measurable index of heart parasympathetic activity. The post - exercise heart rate recovery is computed as peak heart rate minus the heart rate measured after 1 min of termination of exercise. The risk of cardiovascular disease in individual with African background is greater as compared to Caucasians and Asian population. The aim of this study was to analyze the difference in post exercise heart rate recovery in individuals from different ethnic backgrounds. Methods: A thorough literature search was carried out on PubMed, Google Scholar and PakMediNet with articles published between 2007 till 2021. The articles with post - exercise heart rate recovery in male adults with different ethnic backgrounds were included. A total of 8 studies were included after meeting the inclusion criteria for the meta-analysis. The studies that measured heart rate recovery after 1 minute of termination of exercise were included.

Results: Out of 174 subjects 51 individuals were Africans, 104 were Asians and 19 were Caucasians. The post exercise heart raterecovery was not found to be statistically significant between the individuals from different ethnic groups ( $\mathrm{p}$ value-0.258). Conclusion: The post - exercise heart rate recovery is not influenced by the racial differences. Further research on larger scale assessing the impact of parasympathetic activity in terms of heart rate recovery in individual from different ethnic background is required as very limited data is available till date.
\end{abstract}

Keywords: PEHRR, Ethnicities, Sedentary adult males, Racial, Exercise

*Correspondence: Sana Soomro, MPhil scholar, Basic Medical Science Institute, Jinnah Postgraduate medical center, Karachi. Tell -923322323907, Email:sana.soomro@gmail.com

\section{Introduction}

The autonomic nervous system controls the various functions of body's viscera; the performance of individual during the exercise depends on the maximum functioning of autonomic nervous system[1]. The autonomic nervous system involves two main division i.e. sympathetic and parasympathetic nervous system [2]. The most important organ system that is studied widely in relation to autonomic physiology is cardiovascular system which involves both sympathetic and parasympathetic activity [3]. The heart rate increases as soon as individual starts exercising representing the intact function of sympathetic nervous system, but as individual stops workout the heart rate starts to decline which signifies the intact parasympathetic system $[4,5]$. When the parasympathetic activity decrease or the sympathetic activity is enhanced, this condition is referred as 'autonomic imbalance' which increases the risk of death due to cardiac diseases [6] as workload on heart is increased which predispose patient at the risk of developing arrhythmias [7]. The heart rate is the most crucial tool for the assessment of intensity of exercise [8]. During the exercise the impulses from higher brain centers travel to the medullary cardiovascular center to change the arterial pressure to the higher level whereby increasing heart rate by deactivating 
parasympathetic activity [9]. The overall adjustment in the heart rate is made to increase cardiac output, which is the product of heart rate to stroke volume, to meet the metabolic demand of the body during exercise ${ }^{3}$. About $20 \%$ of world population lives sedentary life [10], with total 1.26 million deaths occurs as a result of living sedentary life [11]. The sedentary style of living life produces the autonomic imbalance in individual's life [12], with sympathetic activity becoming predominant in physical inactive individuals [13].

Post exercise heart rate recovery (PEHRR) is defined as the decline in the rate of heart after the exercise has been terminated $[14,15]$. PEHRR is the cheap, noninvasive and easily measurable tool which is readily used in clinical settings to estimate the heart parasympathetic activity [16, 17]. Heart rate recovery is the time required to decrease the heart rate after the exercise which denotes the degree of autonomic nervous system activation, with parasympathetic being overriding post exercise [18]. The post - exercise heart rate recovery is calculated as peak heart rate minus the heart rate measured after 1 minute of termination of exercise [16]. Higher PEHRR indicates the physical fitness of individual [19]. Reduced PEHRR measured after the 1 and 2 minutes of exercise is considered as the determinant of parasympathetic dysfunction [20]. Normally the decline in heart rate is about 12 to 22 beats or more after 1 min of exercise in recovery phase, but the decline in heart rate after 2 minutes of exercise is associated with worst cardiovascular outcomes [21]. The heart rate less than 12 beats per minute measured after 1 minute of exercise in recovery phase is considered abnormal [21]. The PEHRR measured at 1st min of exercise termination is considered important marker of heart parasympathetic activity [21]. The slower reduction in heart rate after exercise in recovery phase denotes depressed vagal activity to heart $[22,23]$. Low post - exercise heart rate recovery indicates poor cardiovascular fitness which is generally affected by obesity and metabolic derangement in individual [13] and hence predicts the mortality in the patients with cardiovascular dysfunction[18, 23, 24].

Globally every year large number of individual dies as a result of cardiovascular diseases [25]. Men are more prone to cardiovascular diseases and mortality associated with cardiovascular events as compare to females [26]. The sedentary life style is one of the major risk factor for the development of cardiovascular related diseases [27]. The autonomic functions become abnormal in individuals with cardiovascular disease, with sympathetic system become enhanced and parasympathetic system becomes depressed [28]. African individuals have greater propensity to develop cardiovascular diseases as compared to the white owing to the fact that they tend to be more obese as compared to the Caucasians [29, 30] but Asians as compared to Caucasian have lower prevalence of cardiovascular disease [28].The study by Schutte et al " [29] report that Asians as compared to Caucasians have higher chances of developing cardiovascular disease. The comparison of autonomic recovery after exercise in Africans, Caucasian and Asians has not been studied and if any variability in heart rate recovery exist between the individuals belonging to different racial background.

\section{Methodology:}

This meta-analysis study was conducted in the department of Physiology, Basic medical science Institute, Jinnah postgraduate medical Centre, Karachi. A thorough systemic literature search using electronic data bases of Google Scholar, PubMed and PakMediNet with articles and abstract published in English language was carried out from 2007 till 2021. The published abstracts and articles were searched via aforementioned search engines with the use of following keywords 'Heart rate recovery', 'Asian', 'African', 'Caucasian', 'Europeans', Sedentary and 'Adult males'.

The inclusion criteria for the study were as follows;

1) Studies published between 2007 till 2021

2) Adult males between 18 to 66 years of age

3) Post exercise Heart rate measured after 1 minute of cessation of exercise 
4) Individuals without any cardiovascular, metabolic, pulmonary, musculoskeletal and peripheral vascular diseases

5) Body mass index (BMI) $<25 \mathrm{Kg} / \mathrm{m}^{2}$.

6) Sedentary individuals for last 6 months

7) Subjects from different ethnic backgrounds

5 cross sectional studies in total that met above mentioned inclusion criteria were included in which peak heart rate (PHR) and post exercise heart rate after 1 min of exercise in recovery phase $\left(\mathrm{HR}_{1} \mathrm{~min}\right)$ were measured in male sedentary adults. The subjects were divided into 3 groups according to major ethnic background i.e. Africans, Asians and Caucasians. The post exercise heart rate recovery (PEHRR) was measured using formula Peak heart rate - Postexercise heart rate after 1 min of cessation of exercise in recovery phase [31].

\section{Statistical Analysis}

The data was collected in Microsoft Excel which included mean age, height, weight, BMI, peak exercise heart rate, heart rate measured after $1 \mathrm{~min}$ of termination of exercise in recovery phase, sample size of each study selected for the meta-analysis. The data was analyzed using SPSS version 22. The age and BMI was reported as mean and standard deviation. ANOVA was applied to assess the significant difference between the groups. Results were considered significant with $\mathrm{P}$ value of $<0.05$.

\section{Results}

Out of 174 individuals from the five studies selected, 51 individuals were Africans, 104 were Asians and 19 were Caucasians, included in the meta-analysis study (Fig 1). The African subjects were Nigerians, Caucasians comprised of subjects from Serbia and Asian constituted Indian, Japanese and South Korean.

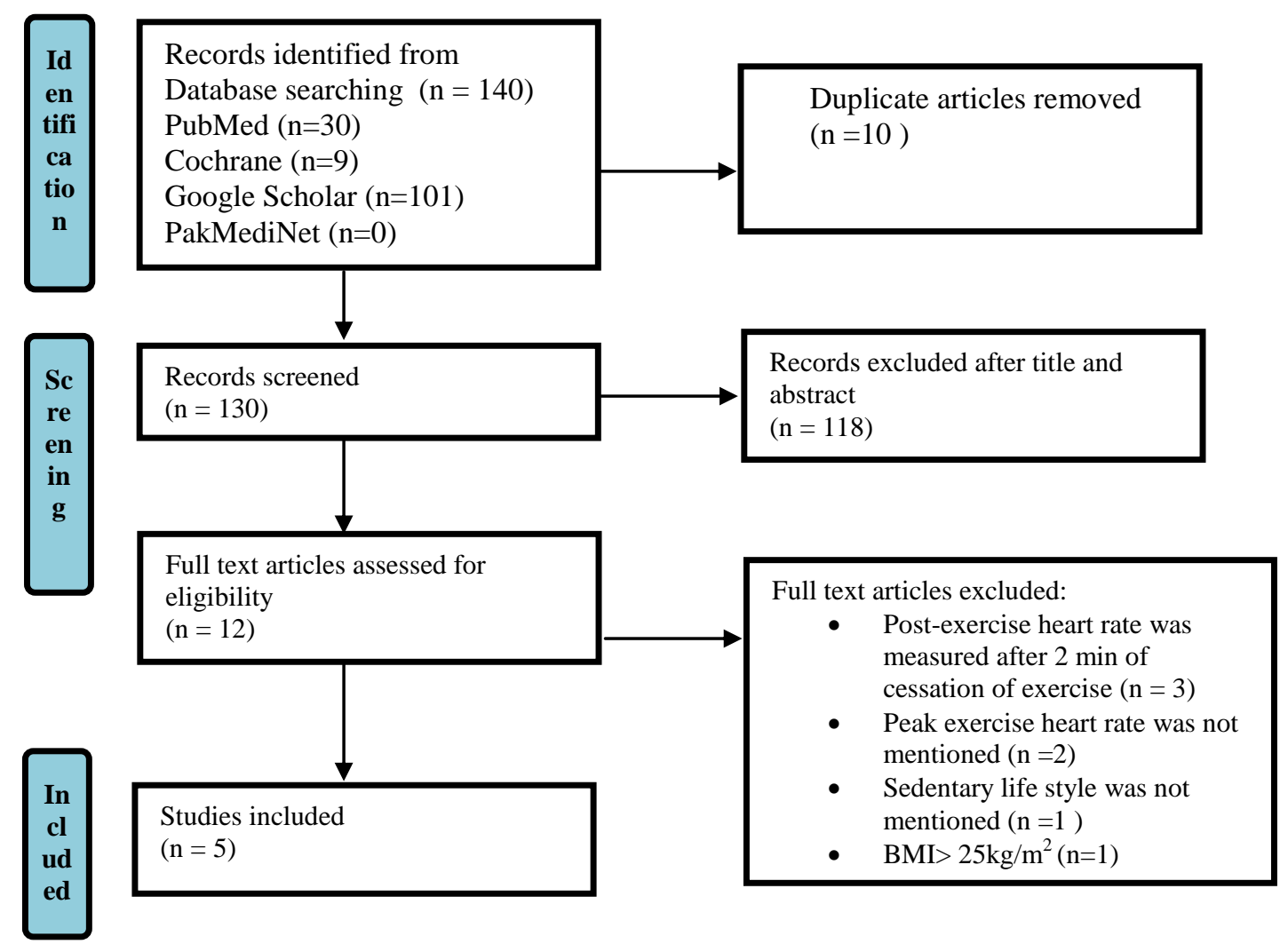

Figure1: PRISMA flow diagram 
Table 1 shows the basic characteristics of studies included in the meta-analysis. The mean age of subjects in the study was lowest $19.9 \pm 1.0$ years to the highest age of $49.1 \pm 5.6$ years. The mean BMI of the study subjects was lowest $21.7 \pm 2.1 \mathrm{~kg} / \mathrm{m}^{2}$ and maximum value of $24.84 \mathrm{~kg} / \mathrm{m}^{2}$.

Table 2 shows the different criteria for estimating sedentary life style, exercise protocol and measurement of heart rate by each study.

Table 3 and Figure 2 shows the different heart rate indices among the various ethnic groups. The Peak heart rate in Caucasians was highest as compared to Africans and Asians with no significant difference found among the different ethnic groups (p-0.063). The decline in heart rate at the end of exercise in recovery phase which is denoted by $\mathrm{HR}_{1}$ min and post exercise Heart Rate Recovery (PEHRR) was found to be more in Caucasian than Asian than African individuals but no significance ( $\mathrm{p}-0.258)$ was noted in terms of heart rate recovery.

Table 1: General Characteristics of Cross-Sectional Studies

\begin{tabular}{|l|l|l|l|l|l|}
\hline Author & $\begin{array}{l}\text { Publication } \\
\text { year }\end{array}$ & $\begin{array}{l}\text { No of } \\
\text { Males }\end{array}$ & $\begin{array}{l}\text { Country of } \\
\text { Origin }\end{array}$ & $\begin{array}{l}\text { Age } \\
(\text { Years })\end{array}$ & $\begin{array}{l}\mathbf{B M I} \\
\mathbf{( K g / \mathbf { m } ^ { 2 }}\end{array}$ \\
\hline Patil [32] & 2012 & 30 & India & $25.9 \pm 2.3$ & $22.5 \pm 1.36$ \\
\hline Barak [33] & 2011 & 19 & Serbia & $19.9 \pm 1.0$ & 24.84 \\
\hline Oyeyemi [13] & 2015 & 51 & Nigeria & $24.84 \pm 3.19$ & $21.85 \pm 3.02$ \\
\hline Kwon [34] & 2014 & 50 & S. Korea & $49.1 \pm 5.6$ & $23.92 \pm 2.0$ \\
\hline Matsuo [35] & 2014 & 12 & Japan & $29.8 \pm 6.9$ & $22.0 \pm 1.7$ \\
\hline Matsuo [36] & 2014 & 12 & Japan & $28.5 \pm 7.7$ & $21.7 \pm 2.1$ \\
\hline
\end{tabular}

Values expressed as mean \pm Standard Deviation

HAIT - high intensity aerobic interval trainingCAT-continuous aerobic training, BMI - body mass index

Table 2: Sedentary criteria and exercise protocol of cross-sectional studies

\begin{tabular}{|l|l|l|l|}
\hline Author & Sedentary criteria & Exercise protocol & Heart rate measurement \\
\hline Patil [32] & - & Harvard step test & Digital electronic device \\
\hline Oyeyemi [13] & $\begin{array}{l}\text { Individual spending 450 } \\
\text { minutes per day in sitting } \\
\text { position }\end{array}$ & $\begin{array}{l}\text { 25 watt Work load } \\
\text { exercise till heart rate } \\
\text { reached 70\% of maximum } \\
\text { heart rate }\end{array}$ & Digital electronic device \\
\hline Barak [33] & $\begin{array}{l}\text { Individual not engaged in } \\
\text { regular physical exercise } \\
\text { for last 6 months }\end{array}$ & $\begin{array}{l}\text { Wingate anaerobic test } \\
\text { minutes for less than 3 } \\
\text { times a week }\end{array}$ & Digital electrocardiogram \\
\hline Kwon [34] & $\begin{array}{l}\text { Individual not engaged in } \\
\text { regular exercise for past } \\
\text { 12 months }\end{array}$ & $\begin{array}{l}3 \text { days a week cycling } \\
\text { HAT- individual VO } \\
\text { max reaching 80\% } \\
\text { CAT-individual } \mathrm{VO}_{2} \mathrm{max}_{2} \\
\text { reaching 60-65\% }\end{array}$ & 12 lead EGG \\
\hline
\end{tabular}

HAIT - High intensity aerobic interval training, CAT - Continuous aerobic training, $\mathrm{VO}_{2 \max }-$ maximal oxygen consumption 
Biosight 2021; 02(02): 58-66

Table 3: PHR, $\mathrm{HR}_{1}$ min and PEHRR among different ethnic groups

\begin{tabular}{|l|l|l|l|l|}
\hline Variable & Africans & Asians & Caucasians & P-value \\
\hline PHR (b/m) & $113.55 \pm 16.56$ & $164.89 \pm 17.69$ & $183.1 \pm 3.1$ & 0.063 \\
\hline HR $\mathbf{1}$ min (b/m) & $96.59 \pm 14.35$ & $138.14 \pm 11.15$ & $153.1 \pm 3.0$ & $0.034^{*}$ \\
\hline PHRRe (b/m) & $16.96 \pm 2.21$ & $26.74 \pm 10.18$ & $30.00 \pm 5.0$ & 0.258 \\
\hline
\end{tabular}

Values expressed as mean \pm Standard Deviation, PHR - Peak heart rate, $\mathrm{HR}_{1} \mathrm{~min}$ - heart rate after 1 minute of exercise, PHHRe - Post exercise heart rate recovery, $\mathrm{b} / \mathrm{m}$ - beats per minute $* \mathrm{P}$ value of $<0.05$ is considered significant

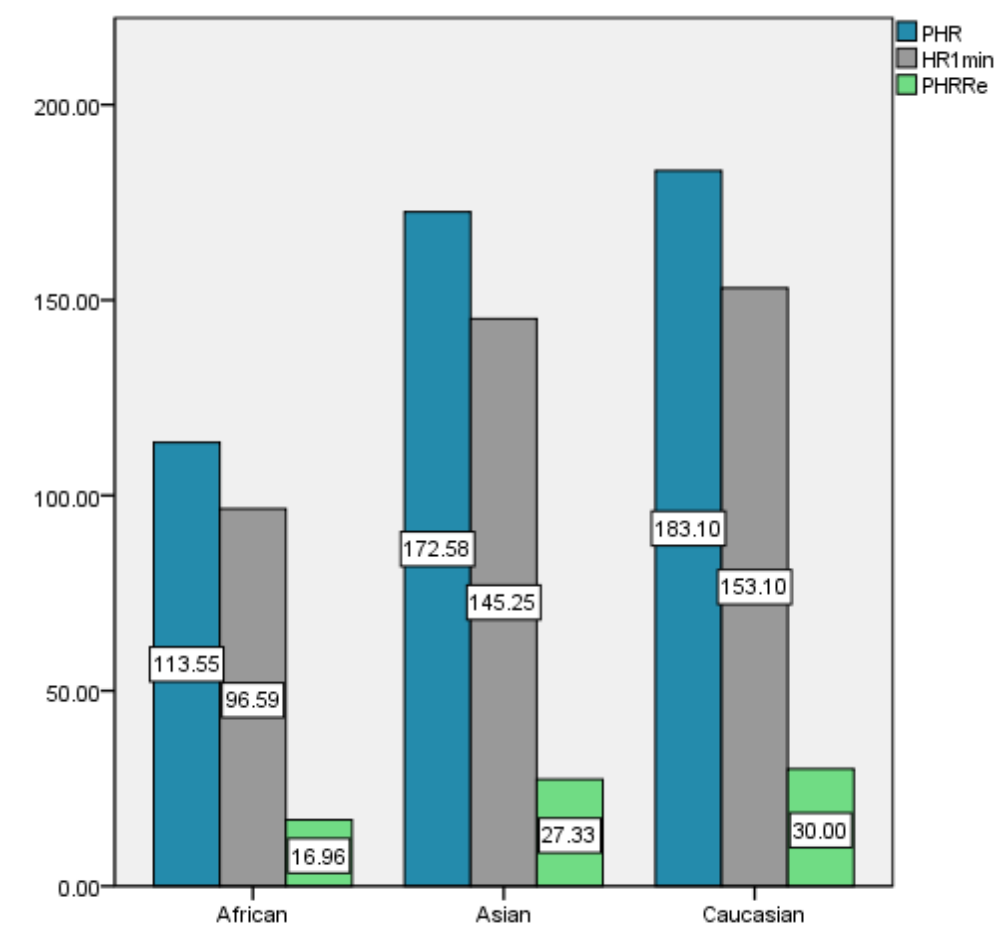

Figure: 2 Variation in heart rate parameters in different ethnic groups

\section{Discussion}

The post exercise heart rate recovery refers to the reduction in the heart rate when the individual has stopped exercising [36]. During exercise the sympathetic arm of autonomic nervous system is predominated which results in increased heart rate, whereby after the exercise is over the parasympathetic arm activates which leads to recovery of heart rate back to baseline [37, 38]. The post exercise heart rate recovery is used clinically to predict future cardiovascular events [14]. Individuals who are not exercisers, die early as compared to individuals who engage themselves in physical activities, with frequent exercises starting as early as individual reaches adulthood save the person from later risk of developing cardiovascular disease [39]. The imbalance in autonomic nervous system functioning occurs frequently in the patients with cardiovascular disease with parasympathetic system becomes depress and sympathetic system becoming enhanced [39, 40]. It is the established fact that heart is mainly regulated by autonomic nervous system which may be influenced by different ethnicities; but the variation in heart autonomic response to exercise in terms of ethnic differences hasn't been studied extensively [41, 42]. The data measuring the parasympathetic activity by heart rate variability is available between different 
ethnic groups but the data of comparative studies on ethnic differences on heart rate recovery is lacking.

The current meta-analysis study included five previous studies on sedentary males to analyze the ethnic differences on heart rate recovery in individuals with normal body mass index. The heart rate recovery in Caucasian was not found to be significant among individuals from different ethnic backgrounds. Although statistically no differences found but the heart rate recovery was observed to be bit higher in Caucasians than Asians and Africans individuals. The findings of this study is consistent with the Esco [30] whereheart rate recoveryin African origin individuals measured 1 minute after the exercise reached maximum intensity was not found to be different as compared to white individuals. The study by Choi et al (2006) [43] on ethnic differences on parasympathetic activity observed the decrease parasympathetic activity in African descendants of America as compared to Caucasian Americans. Also the study by Vranish 2018 [44] O'Neil 2017 [45], Colangelo2020 [46] and Zion 2003 [47] observed that sympathetic system in African origin individuals is enhanced as compared to the white males.The higher arterial stiffness in African origin individuals causes the sustained stretch of afferent fibers decreases the sensitivity of cardioinhibitory area thereby decreasing parasympathetic outflow and increasing sympathetic outflow to heart [44, 47]. The parasympathetic activity in Asians is also lower than the Caucasian [48, 49]. As the studies on autonomic functions of heart has not been studied in detail in Asians and African individuals so the underlying mechanism of autonomic differences on heart activity among individuals from different racial background remains unclear[7, 50]

\section{Concluson}

The current meta-analysis study concludes that post exercise heart rate recovery in healthy sedentary males is not influenced by the ethnic differences. The study on larger scale is required to assess the variation in autonomic activity of heart.

\section{Consent for Publication}

Not applicable.

\section{Standards Of Reporting}

PRISMA guidelines and methodology were followed.

\section{Funding}

None.

\section{Conflict of Interest}

The authors declare no conflict of interest, financial or otherwise.

\section{Acknowledgements}

None.

\section{References}

1. Bisschoff CA, Coetzee B, Esco MR. Heart rate variability and recovery as predictors of elite, African, male badminton players' performance levels. International Journal of Performance Analysis in Sport. 2018 Jan 2;18(1):1-6.

2. Sheng Y, Zhu L. The crosstalk between autonomic nervous system and blood vessels. International journal of physiology, pathophysiology and pharmacology. 2018;10(1):17.

3. Ziemssen T, Siepmann T. The investigation of the cardiovascular and sudomotor autonomic nervous system - a review. Frontiers in neurology. 2019 Feb 12;10:53. 


\section{Biosight 2021; 02(02): 58-66}

4. Daanen HA, Lamberts RP, Kallen VL, Jin A, Van Meeteren NL. A systematic review on heart-rate recovery to monitor changes in training status in athletes. International journal of sports physiology and performance. 2012 Sep 1;7(3):251-60.

5. Azam F, Shaheen A, Irshad K, Liaquat A, Naveed H, Shah SU. Association of postexercise heart rate recovery with body composition in healthy male adults: Findings from Pakistan. Annals of Noninvasive Electrocardiology. 2020 May;25(3):e12711.

6. Jouven X, Empana JP, Schwartz PJ, Desnos M, Courbon D, Ducimetière P. Heart-rate profile during exercise as a predictor of sudden death. New England journal of medicine. 2005 May 12;352(19):1951-8.

7. Lampert R, Ickovics J, Horwitz R, Lee F. Depressed autonomic nervous system function in African Americans and individuals of lower social class: a potential mechanism of race-and classrelated disparities in health outcomes. American heart journal. $2005 \mathrm{Jul}$ 1;150(1):153-60.

8. Prado DM, Dias RG, Trombetta IC. Cardiovascular, ventilatory, and metabolic parameters during exercise: differences between children and adults. Arquivos brasileiros de cardiologia. 2006;87:e149-55.

9. Michael S, Graham KS, Davis GM. Cardiac autonomic responses during exercise and postexercise recovery using heart rate variability and systolic time intervals - a review. Frontiers in physiology. 2017 May 29;8:301.

10. Alansare A, Alford K, Lee S, Church T, Jung HC. The effects of high-intensity interval training vs. moderate-intensity continuous training on heart rate variability in physically inactive adults. International journal of environmental research and public health. $2018 \mathrm{Jul} ; 15(7): 1508$.

11. Memon AR. Physical activity to prevent non-communicable diseases: current status and nationallevel policy in Pakistan. Journal of Pakistan Medical Association. 2020 Jan 14.

12. Espinoza-Salinas A, Molina-Sotomayor E, Cano-Montoya J, Gonzalez-Jurado JA. Is Active Lifestyle Related to Autonomic Nervous System Function and Lipid Profile in People with Overweight? A Study Pilot. Sustainability. 2021 Jan;13(5):2439.

13. Oyeyemi AY, Ewah PA, Oyeyemi AL. Comparison of recovery cardiovascular responses of young physically active and sedentary Nigerian undergraduates following exercise testing. International Journal of Physical Education, Sports and Health. 2015;2(2):60-5.

14. Van de Vegte YJ, van der Harst P, Verweij N. Heart rate recovery 10 seconds after cessation of exercise predicts death. Journal of the American Heart Association. 2018 Apr 5;7(8):e008341.

15. Dimkpa U, Oji JO. Association of heart rate recovery after exercise with indices of obesity in healthy, non-obese adults. European journal of applied physiology. 2010 Mar;108(4):695-9.

16. Buchheit, M., Papelier, Y., Laursen, P. B., \& Ahmaidi, S. (2007). Noninvasive assessment of cardiac parasympathetic function: postexercise heart rate recovery or heart rate variability? American Journal of Physiology-Heart and Circulatory Physiology, 293(1), H8-H10.

17. Pepera G, Panagiota Z. Comparison of heart rate response and heart rate recovery after step test among smoker and non-smoker athletes. African Health Sciences. 2021 Apr 16;21(1):105-1.

18. Yu, Y., Liu, T., Wu, J., Zhu, P., Zhang, M., Zheng, W., \& Gu, Y. (2017). Heart rate recovery in hypertensive patients: relationship with blood pressure control. Journal of human hypertension, 31(5), 354-360.

19. Wändell, P. E., Carlsson, A. C., \& Theobald, H. (2010). Effect of heart-rate recovery on long-term mortality among men and women. International journal of cardiology, 144(2), 276-279.

20. Carroll, S., Marshall, P., Ingle, L., \& Borkoles, E. (2012). Cardiorespiratory fitness and heart rate recovery in obese premenopausal women. Scandinavian journal of medicine \& science in sports, 22(6), e133-e139.

21. Morita AA, Silva LK, Bisca GW, Oliveira JM, Hernandes NA, Pitta F, Furlanetto KC. Heart rate recovery, physical activity level, and functional status in subjects with COPD. Respiratory care. 2018 Aug 1;63(8):1002-8.

22. Guilkey JP, Dykstra B, Erichsen J, Mahon AD. Heart rate response and variability following maximal exercise in overweight children. Pediatric exercise science. 2017 Aug 1;29(3):341-9.

23. Hattiwale HM, Hattiwale SH, Dhundasi SA, Das KK. Recovery heart rate response in sedentary and physically active young healthy adults of Bijapur, Karnataka, India. Basic Sci Med. 2012;1(5):30-3. 


\section{Biosight 2021; 02(02): 58-66}

24. Facioli TP, Philbois SV, Gastaldi AC, Almeida DS, Maida KD, Rodrigues JA, Sánchez-Delgado JC, Souza HC. Study of heart rate recovery and cardiovascular autonomic modulation in healthy participants after submaximal exercise. Scientific Reports. 2021 Feb 11;11(1):1-9.

25. Al-Shamsi S, Regmi D, Govender RD. Incidence of cardiovascular disease and its associated risk factors in at-risk men and women in the United Arab Emirates: a 9-year retrospective cohort study. BMC cardiovascular disorders. 2019 Dec;19(1):1-9.

26. Koenig J, Thayer JF. Sex differences in healthy human heart rate variability: a meta-analysis. Neuroscience \& Biobehavioral Reviews. 2016 May 1;64:288-310.

27. Vasankari V, Husu P, Vähä-Ypyä H, Suni J, Tokola K, Halonen J, Hartikainen J, Sievänen H, Vasankari T. Association of objectively measured sedentary behaviour and physical activity with cardiovascular disease risk. European journal of preventive cardiology. 2017 Aug 1;24(12):13118.

28. Sun P, Yan H, Ranadive SM, Lane AD, Kappus RM, Bunsawat K, Baynard T, Hu M, Li S, Fernhall B. Autonomic recovery is delayed in Chinese compared with Caucasian following treadmill exercise. PloS one. 2016 Jan 19;11(1):e0147104.

29. Schutte AE. Ethnicity and Cardiovascular Disease. InTextbook of Vascular Medicine 2019 (pp. 287-295). Springer, Cham.

30. Esco M. Cardiovascular autonomic modulation following maximal exercise: Its relationship to race, $\mathrm{VO} 2 \mathrm{max}$, and resting heart rate variability (Doctoral dissertation)

31. Medeiros, W. M., de Luca, F. A., de Figueredo Júnior, A. R., Mendes, F. A., \& Gun, C. (2018). Heart rate recovery improvement in patients following acute myocardial infarction: exercise training, $\beta$-blocker therapy or both. Clinical physiology and functional imaging, 38(3), 351-359.

32. Patil RB, Doddamani BR, Bhutkar M, Awanti SM. A comparative study of physical fitness among rural farmers and urban sedentary group of Gulbarga District. Al. Ameen J. Med. Sci. 2012;5(1):39-44.

33. Barak OF, Ovcin ZB, Jakovljevic DG, Lozanov-Crvenkovic Z, Brodie DA, Grujic NG. Heart rate recovery after submaximal exercise in four different recovery protocols in male athletes and nonathletes. Journal of sports science \& medicine. 2011 Jun;10(2):369.

34. Kwon O, Park S, Kim YJ, Min SY, Kim YR, Nam GB, Choi KJ, Kim YH. The exercise heart rate profile in master athletes compared to healthy controls. Clinical physiology and functional imaging. $2016 \mathrm{Jul} ; 36(4): 286-92$.

35. Matsuo T, Saotome K, Seino S, Eto M, Shimojo N, Matsushita A, Iemitsu M, Ohshima H, Tanaka K, Mukai C. Low-volume, high-intensity, aerobic interval exercise for sedentary adults: $\$$ \$ ldot $\{\mathrm{V}\} \$ \$ \mathrm{~V}^{\cdot} \mathrm{O} 2 \mathrm{max}$, cardiac mass, and heart rate recovery. European journal of applied physiology. 2014 Sep;114(9):1963-72.

36. Qiu S, Cai X, Sun Z, Li L, Zuegel M, Steinacker JM, Schumann U. Heart rate recovery and risk of cardiovascular events and all-cause mortality: a meta-analysis of prospective cohort studies. Journal of the American Heart Association. 2017 May 9;6(5):e005505.

37. Kokkinos P, Myers J, Doumas M, Faselis C, Pittaras A, Manolis A, Kokkinos JP, Narayan P, Papademetriou V, Fletcher R. Heart rate recovery, exercise capacity, and mortality risk in male veterans. European journal of preventive cardiology. 2012 Apr 1;19(2):177-84.

38. Rahul NV, Agarwal M, Vishwakarma P, Kanchan A, Kumar P. Heart rate recovery in normal and obese males with and without parental history of cardiovascular disease. Journal of Family Medicine and Primary Care. 2020 May;9(5):2379.

39. Fan LM, Collins A, Geng L, Li JM. Impact of unhealthy lifestyle on cardiorespiratory fitness and heart rate recovery of medical science students. BMC public health. 2020 Dec;20(1):1-8.

40. Peçanha T, Silva-Júnior ND, Forjaz CL. Heart rate recovery: autonomic determinants, methods of assessment and association with mortality and cardiovascular diseases. Clinical physiology and functional imaging. 2014 Sep;34(5):327-39.

41. Baek, J., Park, D., Kim, I., Won, J. U., Hwang, J., \& Roh, J. (2013). Autonomic dysfunction of overweight combined with low muscle mass. Clinical Autonomic Research, 23(6), 325-331.

42. Derevere J. Effect of Ethnicity on Changes in VO2max and Cardiac Output in Response to ShortTerm High Intensity Interval Training. 


\section{Biosight 2021; 02(02): 58-66}

43. Choi JB, Hong S, Nelesen R, Bardwell WA, Natarajan L, Schubert C, Dimsdale JE. Age and ethnicity differences in short-term heart-rate variability. Psychosomatic medicine. 2006 May 1;68(3):421-6.

44. Vranish JR, Holwerda SW, Young BE, Credeur DP, Patik JC, Barbosa TC, Keller DM, Fadel PJ. Exaggerated vasoconstriction to spontaneous bursts of muscle sympathetic nerve activity in healthy young black men. Hypertension. 2018 Jan;71(1):192-8.

45. Colangelo LA, Yano Y, Jacobs Jr DR, Lloyd-Jones DM. Association of Resting Heart Rate With Blood Pressure and Incident Hypertension Over 30 Years in Black and White Adults: The CARDIA Study. Hypertension. 2020 Sep;76(3):692-8.

46. O'Neill J, Tayebjee MH. Why are South Asians seemingly protected against the development of atrial fibrillation? A review of current evidence. Trends in cardiovascular medicine. 2017 May 1;27(4):249-57.

47. Zion AS, Bond V, Adams RG, Williams D, Fullilove RE, Sloan RP, Bartels MN, Downey JA, De Meersman RE. Low arterial compliance in young African-American males. American Journal of Physiology-Heart and Circulatory Physiology. 2003 Aug;285(2):H457-62.

48. Chaturvedi, N. (2003). Ethnic differences in cardiovascular disease. Heart, 89(6), 681-686.

49. Bathula R, Hughes AD, Panerai R, Potter J, Thom SM, Francis DP, Shore AC, Kooner J, Chaturvedi N. Indian Asians have poorer cardiovascular autonomic function than Europeans: this is due to greater hyperglycaemia and may contribute to their greater risk of heart disease. Diabetologia. 2010 Oct;53(10):2120-8.

50. Lau CP, Tse HF, Siu CW, Gbadebo D. Atrial electrical and structural remodeling: implications for racial differences in atrial fibrillation. J Cardiovasc Electrophysiol. 2012 Nov;23 Suppl 1:S36-40. doi: 10.1111/jce.12022. PMID: 23140346. 